\title{
CONVERGENCE OF ADOMIAN DECOMPOSITION METHOD FOR PDES
}

\author{
SILVIA SEMINARA ${ }^{\dagger}$ AND MARIA INÉS TROPAREVSKY
}

\author{
Date of Receiving : $\quad$ : 25.06.2015 \\ Date of Revision : $\quad 06.10 .2015$ \\ Date of Acceptance : $\quad 06.10 .2015$
}

\begin{abstract}
Adomian Decomposition Method (ADM) is a powerful tool for solving general functional equations. It provides a sequence of analytic approximate solutions to a wide range of nonlinear problems. Trying to formulate a general framework for the method, several demonstrations of convergence have been published, with varying degree of success. In this work we state conditions under which the approximate solutions to partial differential equations provided by ADM are merely a reordering of the Taylor polynomials of the solution, and, consequently, no other demonstration of convergence is necessary in those cases.
\end{abstract}

\section{Introduction}

Adomian Decomposition Method (ADM) was introduced by George Adomian in the '80s ([2], [3], [4]). It provides analytical approximate solutions for very general nonlinear equations without linearization or simplification. The description of the method is simple, but, because of the wide range of problems it covers, it is difficult to state a general demonstration of convergence. The author gave no formal proofs but provided (without showing the details) a justification based on the composition of Taylor series. He offered lots of examples of application to problems with well known solutions, to show the goodness of the method. In the last two decades, a great quantity of works have been published where it was successfully applied to many different problems. More or less general demonstrations of convergence were also offered.

In this work we state conditions under which the solutions provided by ADM to certain partial differential equations (PDE) are just reorderings of the Taylor polynomials of the solution, as George Adomian posited. We formally prove this issue and show some examples.

Key words and phrases. Nonlinear equations, Approximate analytical solutions, Adomian decomposition method, PDEs.

Communicated by. Virender

${ }^{\dagger}$ Corresponding author. 